\title{
Hyers-Ulam stability of an alternative functional equation of Jensen type
}

\section{Choodech Srisawat}

Department of Mathematics, Faculty of Science, Udon Thani Rajabhat University, Udon Thani 41000 Thailand

e-mail: ch.srisawat@gmail.com

Received 6 Jul 2018

Accepted 30 Jun 2019

ABSTRACT: Given an integer $\lambda \notin\{-2,-1,0,2\}$, we investigate the Hyers-Ulam stability of an alternative Jensen's functional equation $f\left(x y^{-1}\right)-2 f(x)+f(x y)=0$ or $f\left(x y^{-1}\right)-\lambda f(x)+f(x y)=0$ where $f$ is a mapping from an abelian group to a Banach space.

KEYWORDS: stability, alternative equation, Jensen's functional equation

MSC2010: 39B82 39B72

\section{INTRODUCTION}

The problem of the alternative Cauchy functional equation has been widely studied. For instance, Kannappan et $\mathrm{al}^{1}$ studied the solutions of the alternative Cauchy functional equation of the form

$$
\begin{aligned}
& (f(x+y)-a f(x)-b f(y)) \\
& (f(x+y)-f(x)-f(y))=0,
\end{aligned}
$$

where $f$ is a function from an abelian group to a commutative integral domain of characteristic zero. $\mathrm{Ger}^{2}$ extended their results to the alternative functional equation

$$
\begin{aligned}
(f(x+y)-a f(x)-b f(y)) & \\
(f(x+y)-c f(x)-\mathrm{d} f(y)) & =0 .
\end{aligned}
$$

Forti ${ }^{3}$ found the general solution of the alternative Cauchy functional equation of the form

$$
\begin{aligned}
(c f(x+y)-a f & (x)-b f(y)-d) \\
& (f(x+y)-f(x)-f(y))=0 .
\end{aligned}
$$

Nakmahachalasint ${ }^{4}$ first solved an alternative Jensen's functional equation of the form

$$
f(x) \pm 2 f(x y)+f\left(x y^{2}\right)=0
$$

on a semigroup. His research extended the work of $\mathrm{Ng}^{5}$ and Parnami et $\mathrm{al}^{6}$ on the classical Jensen's functional equation

$$
f\left(x y^{-1}\right)-2 f(x)+f(x y)=0
$$

on a group. Nakmahachalasint ${ }^{7}$ then investigated the Hyers-Ulam stability of the alternative Jensen's functional equation (2) in the class of mappings from 2-divisible abelian groups to Banach spaces.

Given an integer $\lambda \neq 2$, Srisawat et $\mathrm{al}^{8}$ solved the alternative Jensen's functional equation of the form

$$
\begin{aligned}
& f\left(x y^{-1}\right)-2 f(x)+f(x y)=0 \quad \text { or } \\
& f\left(x y^{-1}\right)-\lambda f(x)+f(x y)=0
\end{aligned}
$$

when $f$ is a function from a group to a uniquely divisible abelian group, but a stability problem has not yet been investigated. In this paper, we will prove the Hyers-Ulam stability of the alternative Jensen's functional equation (4) when $\lambda \notin\{-2,-1,0,2\}$ is an integer and $f$ is a mapping from an abelian group $(G, \cdot)$ to a Banach space $(E,\|\cdot\|)$. In other words, for every $\varepsilon \geqslant 0$, we prove that there exist $\delta_{1}, \delta_{2} \geqslant 0$ such that if, for an integer $\lambda \notin\{-2,-1,0,2\}$, a mapping $f: G \rightarrow E$ satisfies the inequalities

$$
\begin{aligned}
& \left\|f\left(x y^{-1}\right)-2 f(x)+f(x y)\right\| \leqslant \delta_{1} \quad \text { or } \\
& \left\|f\left(x y^{-1}\right)-\lambda f(x)+f(x y)\right\| \leqslant \delta_{2}
\end{aligned}
$$

for every $x, y \in G$, then there exists a unique Jensen's mapping $J: G \rightarrow E$ with $\|f(x)-J(x)\| \leqslant \varepsilon$ for all $x \in G$.

\section{AUXILIARY LEMMAS}

Throughout this study, we let $(G, \cdot)$ be a group and $(E,\|\cdot\|)$ be a Banach space. Given an integer $\lambda$, and 
a function $f: G \rightarrow E$. For $x, y \in G$, we define

$$
\mathscr{F}_{y}^{(\lambda)}(x):=\left\|f\left(x y^{-1}\right)-\lambda f(x)+f(x y)\right\| .
$$

Furthermore, for $\delta_{1}, \delta_{2} \geqslant 0$ and $\lambda \notin\{-2,-1,0,2\}$, we let

$$
\begin{aligned}
& \mathscr{S} f_{y}^{(\lambda)}(x):=\left(\mathscr{F}_{y}^{(2)}(x) \leqslant \delta_{1} \text { or } \mathscr{F}_{y}^{(\lambda)}(x) \leqslant \delta_{2}\right), \\
& \delta:=\max \left\{\delta_{1}, \delta_{2}\right\}, \quad \text { and } \\
& \mathscr{M}_{\delta}^{\lambda}:=\left(29+42|\lambda|+38 \lambda^{2}+20\left|\lambda^{3}\right|+4 \lambda^{4}\right) \delta .
\end{aligned}
$$

The set of all solution of (5) is denoted by

$$
\mathscr{A}_{(G, E)}^{(\lambda)}:=\left\{f: G \rightarrow E \mid \mathscr{S} f_{y}^{(\lambda)}(x), \forall x, y \in G\right\} .
$$

We first prove the bound of $f(x)$ concerning the relation between $\mathscr{S} f_{y}^{(\lambda)}\left(x y^{-1}\right), \mathscr{S} f_{y}^{(\lambda)}(x)$, and $\mathscr{S} f_{y}^{(\lambda)}(x y)$.

Lemma 1 Let $f \in \mathscr{A}_{(G, E)}^{(\lambda)}$ and $x, y \in G$.

(i) If $\mathscr{F}_{y}^{(2)}\left(x y^{-1}\right) \leqslant \delta_{1}, \mathscr{F}_{y}^{(\lambda)}(x) \leqslant \delta_{2}$, and $\mathscr{F}_{y}^{(2)}(x y) \leqslant \delta_{1}$, then $\|f(x)\| \leqslant 5 \delta$.

(ii) If $\mathscr{F}_{y}^{(\lambda)}\left(x y^{-1}\right) \leqslant \delta_{2}, \quad \mathscr{F}_{y}^{(\lambda)}(x) \leqslant \delta_{2}$, and $\mathscr{F}_{y}^{(\lambda)}(x y) \leqslant \delta_{2}$, then $\|f(x)\| \leqslant(3+|\lambda|) \delta$.

Proof:

(i) We observe that

$$
\begin{aligned}
& \left\|f\left(x y^{-2}\right)+2(1-\lambda) f(x)+f\left(x y^{2}\right)\right\| \\
& \quad \leqslant \mathscr{F}_{y}^{(2)}\left(x y^{-1}\right)+2 \mathscr{F}_{y}^{(\lambda)}(x)+\mathscr{F}_{y}^{(2)}(x y) \\
& \quad \leqslant 4 \delta .
\end{aligned}
$$

Consider the alternatives in $\mathscr{S}_{y^{2}}^{(\lambda)}(x)$. The inequality $\mathscr{F}_{y^{2}}^{(2)}(x) \leqslant \delta_{1}$ and (6) give $\|(4-$ $2 \lambda) f(x) \| \leqslant 5 \delta$, while the inequality $\mathscr{F}_{y^{2}}^{(\lambda)}(x) \leqslant$ $\delta_{2}$ and (6) give $\|(2-\lambda) f(x)\| \leqslant 5 \delta$. Hence $\|f(x)\| \leqslant 5 \delta$.

(ii) We observe that

$$
\begin{aligned}
& \left\|f\left(x y^{-2}\right)+\left(2-\lambda^{2}\right) f(x)+f\left(x y^{2}\right)\right\| \\
& \quad \leqslant \mathscr{F}_{y}^{(\lambda)}\left(x y^{-1}\right)+\lambda \mathscr{F}_{y}^{(\lambda)}(x)+\mathscr{F}_{y}^{(\lambda)}(x y) \\
& \quad \leqslant(2+|\lambda|) \delta .
\end{aligned}
$$

Consider the alternatives in $\mathscr{S} f_{y^{2}}^{(\lambda)}(x)$. The inequality $\mathscr{F}_{y^{2}}^{(2)}(x) \leqslant \delta_{1}$ and (7) give $\|(4-$ $\left.\lambda^{2}\right) f(x) \| \leqslant(3+|\lambda|) \delta$, while the inequality $\mathscr{F}_{y^{2}}^{(\lambda)}(x) \leqslant \delta_{2}$ and (7) give $\left\|\left(2+\lambda-\lambda^{2}\right) f(x)\right\| \leqslant$ $(3+|\lambda|) \delta$. Hence $\|f(x)\| \leqslant(3+|\lambda|) \delta$.
Lemma 2 Let $f \in \mathscr{A}_{(G, E)}^{(\lambda)}$ and $x, y \in G$. If $\mathscr{F}_{y}^{(2)}\left(x y^{-1}\right) \leqslant \delta_{1}, \mathscr{F}_{y}^{(\lambda)}(x) \leqslant \delta_{2}$, and $\mathscr{F}_{y}^{(\lambda)}(x y) \leqslant$ $\delta_{2}$, then $\|f(x)\| \leqslant\left(14+14|\lambda|+12 \lambda^{2}+4|\lambda|^{3}\right) \delta$.

Proof: By $\mathscr{F}_{y}^{(2)}\left(x y^{-1}\right) \leqslant \delta_{1}$ and $\mathscr{F}_{y}^{(\lambda)}(x) \leqslant \delta_{2}$, we obtain

$$
\left\|f\left(x y^{-2}\right)+(1-2 \lambda) f(x)+2 f(x y)\right\| \leqslant 3 \delta .
$$

Next, we consider two possible cases in $\mathscr{S}_{y^{2}}^{(\lambda)}(x)$ :

Case (i): Assume that $\mathscr{F}_{y^{2}}^{(2)}(x) \leqslant \delta_{1}$. Using $\mathscr{F}_{y}^{(\lambda)}(x y) \leqslant \delta_{2}, \mathscr{F}_{y^{2}}^{(2)}(x) \leqslant \delta_{1}$, and (8) we obtain

$$
\|2 f(x)+f(x y)\| \leqslant 5 \delta
$$

and

$$
\left\|(2 \lambda+1) f(x)+f\left(x y^{2}\right)\right\| \leqslant(2+4|\lambda|) \delta .
$$

Eliminating $f(x y)$ from (9) and the alternatives in $\mathscr{S} f_{y}^{(\lambda)}\left(x y^{2}\right)$, we have

$$
\begin{aligned}
& \left\|2 f(x)+2 f\left(x y^{2}\right)-f\left(x y^{3}\right)\right\| \leqslant 6 \delta \quad \text { or } \\
& \left\|2 f(x)+\lambda f\left(x y^{2}\right)-f\left(x y^{3}\right)\right\| \leqslant 6 \delta .
\end{aligned}
$$

By (10) and (11), we obtain

$$
\begin{aligned}
\left\|4 \lambda f(x)+f\left(x y^{3}\right)\right\| & \leqslant(10+8|\lambda|) \delta \quad \text { or } \\
\left\|\left(2 \lambda^{2}+\lambda-2\right) f(x)+f\left(x y^{3}\right)\right\| & \leqslant\left(6+2|\lambda|+4 \lambda^{2}\right) \delta .
\end{aligned}
$$

Consider the alternatives in $\mathscr{S} f_{y^{2}}^{(\lambda)}(x y)$.

(i) If $\mathscr{F}_{y^{2}}^{(2)}(x y) \leqslant \delta_{1}$, then we use $\mathscr{F}_{y^{2}}^{(2)}(x y) \leqslant \delta_{1}$ and $\mathscr{F}_{y}^{(\lambda)}(x) \leqslant \delta_{2}$ to obtain

$$
\left\|\lambda f(x)-3 f(x y)+f\left(x y^{3}\right)\right\| \leqslant 2 \delta .
$$

By (12) and (13), we obtain

$$
\begin{aligned}
\|3 \lambda f(x)+3 f(x y)\| & \leqslant(12+8|\lambda|) \delta \quad \text { or } \\
\left\|\left(2 \lambda^{2}-2\right) f(x)+3 f(x y)\right\| & \leqslant\left(8+2|\lambda|+4 \lambda^{2}\right) \delta .
\end{aligned}
$$

Eliminating $f(x y)$ from (9) and (14), we have $\|f(x)\| \leqslant\left(12+|\lambda|+2 \lambda^{2}\right) \delta$.

(ii) If $\mathscr{F}_{y^{2}}^{(\lambda)}(x y) \leqslant \delta_{2}$, then we use $\mathscr{F}_{y^{2}}^{(\lambda)}(x y) \leqslant \delta_{2}$ and $\mathscr{F}_{y}^{(\lambda)}(x) \leqslant \delta_{2}$ to obtain

$$
\left\|\lambda f(x)-(\lambda+1) f(x y)+f\left(x y^{3}\right)\right\| \leqslant 2 \delta .
$$

By (12) and (15), we obtain

$$
\|3 \lambda f(x)+(\lambda+1) f(x y)\| \leqslant(12+8|\lambda|) \delta \text { or }
$$

$\left\|\left(2 \lambda^{2}-2\right) f(x)+(\lambda+1) f(x y)\right\| \leqslant\left(8+2|\lambda|+4 \lambda^{2}\right) \delta$. 
Eliminating $f(x y)$ from (9) and (16), we obtain $\|f(x)\| \leqslant\left(17+11|\lambda|+2 \lambda^{2}\right) \delta$.

Case (ii). Assume that $\mathscr{F}_{y^{2}}^{(\lambda)}(x) \leqslant \delta_{2}$. Using $\mathscr{F}_{y}^{(\lambda)}(x y) \leqslant \delta_{2}, \mathscr{F}_{y^{2}}^{(\lambda)}(x) \leqslant \delta_{2}$, and (8) we obtain

$$
\|f(x)+f(x y)\| \leqslant 5 \delta
$$

and

$$
\left\|(\lambda+1) f(x)+f\left(x y^{2}\right)\right\| \leqslant(2+4|\lambda|) \delta .
$$

Eliminating $f\left(x y^{2}\right)$ from (18) and the alternatives in $\mathscr{S} f_{y^{2}}^{(\lambda)}\left(x y^{2}\right)$, we obtain

$$
\begin{gathered}
\left\|(2 \lambda+3) f(x)+f\left(x y^{4}\right)\right\| \leqslant(5+8|\lambda|) \delta \quad \text { or } \\
\left\|\left(\lambda^{2}+\lambda+1\right) f(x)+f\left(x y^{4}\right)\right\| \leqslant\left(1+2|\lambda|+4 \lambda^{2}\right) \delta .
\end{gathered}
$$

By (17) and the alternatives in $\mathscr{S} f_{y}^{(\lambda)}\left(x y^{2}\right)$, we have

$$
\begin{aligned}
& \left\|f(x)+2 f\left(x y^{2}\right)-f\left(x y^{3}\right)\right\| \leqslant 6 \delta \quad \text { or } \\
& \left\|f(x)+\lambda f\left(x y^{2}\right)-f\left(x y^{3}\right)\right\| \leqslant 6 \delta .
\end{aligned}
$$

Consider the alternatives in $\mathscr{S} f_{y}^{(\lambda)}\left(x y^{3}\right)$ as follows. (i) If $\mathscr{F}_{y}^{(2)}\left(x y^{3}\right) \leqslant \delta_{1}$, then we eliminate $f\left(x y^{3}\right)$ from (20) and $\mathscr{F}_{y}^{(2)}\left(x y^{3}\right) \leqslant \delta_{1}$ to obtain

$$
\begin{aligned}
& \left\|2 f(x)+3 f\left(x y^{2}\right)-f\left(x y^{4}\right)\right\| \leqslant 13 \delta \text { or } \\
& \left\|2 f(x)+(2 \lambda-1) f\left(x y^{2}\right)-f\left(x y^{4}\right)\right\| \leqslant 13 \delta .
\end{aligned}
$$

Using (18) and (21), we obtain

$$
\begin{aligned}
\left\|(3 \lambda+1) f(x)+f\left(x y^{4}\right)\right\| & \leqslant(19+12|\lambda|) \delta \quad \text { or } \\
\left\|\left(2 \lambda^{2}+\lambda-3\right) f(x)+f\left(x y^{4}\right)\right\| & \leqslant\left(15+8|\lambda|+8 \lambda^{2}\right) \delta .
\end{aligned}
$$

By (19) and (22), we conclude that

$$
\|f(x)\| \leqslant\left(24+12|\lambda|+12 \lambda^{2}\right) \delta .
$$

(ii) If $\mathscr{F}_{y}^{(\lambda)}\left(x y^{3}\right) \leqslant \delta_{2}$, then we eliminate $f\left(x y^{3}\right)$ from (20) and $\mathscr{F}_{y}^{(\lambda)}\left(x y^{3}\right) \leqslant \delta_{2}$ to obtain

$\left\|\lambda f(x)+(2 \lambda-1) f\left(x y^{2}\right)-f\left(x y^{4}\right)\right\| \leqslant(1+6|\lambda|) \delta$ or $\left\|\lambda f(x)+\left(\lambda^{2}-1\right) f\left(x y^{2}\right)-f\left(x y^{4}\right)\right\| \leqslant(1+6|\lambda|) \delta$.

Using (18) and (23), we obtain

$$
\begin{aligned}
& \left\|\left(2 \lambda^{2}-1\right) f(x)+f\left(x y^{4}\right)\right\| \leqslant\left(3+14|\lambda|+8 \lambda^{2}\right) \delta \quad \text { or } \\
& \left\|\left(\lambda^{3}+\lambda^{2}-2 \lambda-1\right) f(x)+f\left(x y^{4}\right)\right\| \\
& \leqslant\left(3+10|\lambda|+2 \lambda^{2}+4\left|\lambda^{3}\right|\right) \delta .
\end{aligned}
$$

By (19) and (24), we conclude that

$$
\|f(x)\| \leqslant\left(8+14|\lambda|+12 \lambda^{2}+4\left|\lambda^{3}\right|\right) \delta 4 .
$$

The desired bound of $f(x)$ follows from the consideration of all cases.

The following lemma is crucial for the main theorem in the next section.
Lemma 3 If $f \in \mathscr{A}_{(G, E)}^{(\lambda)}$, then $\mathscr{F}_{y}^{(2)}(x) \leqslant \mathscr{M}_{\delta}^{\lambda}$ for all $x, y \in G$.

Proof: Let $f \in \mathscr{A}_{(G, E)}^{(\lambda)}$ and $x, y \in G$. Suppose $\mathscr{F}_{y}^{(2)}(x)>\delta_{1}$. From the alternatives in $\mathscr{S} f_{y}^{(\lambda)}(x)$, we obtain $\mathscr{F}_{y}^{(\lambda)}(x) \leqslant \delta_{2}$. The alternatives in $\mathscr{S} f_{y}^{(\lambda)}\left(x y^{-1}\right)$ will be considered as follows.

Case (i). Assume that $\mathscr{F}_{y}^{(2)}\left(x y^{-1}\right) \leqslant \delta_{1}$. By Lemma 1 and Lemma 2, we conclude that

$$
\|f(x)\| \leqslant\left(14+14|\lambda|+12 \lambda^{2}+4\left|\lambda^{3}\right|\right) \delta .
$$

Using $\mathscr{F}_{y}^{(\lambda)}(x) \leqslant \delta_{2}$ and (3), we obtain

$$
\begin{aligned}
& \left\|f\left(x y^{-1}\right)+f(x y)\right\| \\
& \quad \leqslant\left(1+14|\lambda|+14 \lambda^{2}+12\left|\lambda^{3}\right|+4 \lambda^{4}\right) \delta .
\end{aligned}
$$

Hence, by (25) and (26), we have $\mathscr{F}_{y}^{(2)}(x) \leqslant \mathscr{M}_{\delta}^{\lambda}$ as desired.

Case (ii). Assume that $\mathscr{F}_{y}^{(\lambda)}\left(x y^{-1}\right) \leqslant \delta_{2}$. Consider the alternatives in $\mathscr{S} f_{y}^{(\lambda)}(x y)$. If $\mathscr{F}_{y}^{(\lambda)}(x y) \leqslant$ $\delta_{2}$, then Lemma 1 gives $\|f(x)\| \leqslant(3+|\lambda|) \delta$. Thus the desired proof is similar to the above case. If $\mathscr{F}_{y}^{(2)}(x y) \leqslant \delta_{1}$, then the proof is as in case (i) after replacing $y$ by $y^{-1}$ and $x$ by $x y^{-1}$.

\section{HYERS-ULAM STABILITY}

It should be remarked that Srisawat et $\mathrm{al}^{8}$ proved that when $\lambda \notin\{-2,-1,0\}$, the alternative Jensen's functional equation (4) is equivalent to Jensen's functional equation (3). On the other hand, when $\lambda \in\{-2,-1,0\}$, (4) is not necessarily equivalent to (3). In this section, we will prove the HyersUlam stability of the alternative Jensen's functional equation (4) when $\lambda \notin\{-2,-1,0,2\}$ is an integer by the so-called direct method. The stability results of Jensen's functional equation can be found in, for instance, Kominek ${ }^{9}$ or Jung ${ }^{10}$.

Theorem 1 Let $\tilde{G}$ be an abelian group. If $f \in \mathscr{A}_{(\tilde{G}, E)}^{(\lambda)}$, then there exists a unique Jensen's mapping $J: \tilde{G} \rightarrow E$ satisfying (3) with $J(0)=f(0)$ such that $\| f(x)-$ $J(x) \| \leqslant 2 \mathscr{M}_{\delta}^{\lambda}$ for all $x \in \tilde{G}$. Furthermore, the mapping $J$ is given by

$$
J(x)=f(0)+\lim _{n \rightarrow \infty} \frac{1}{2^{n}}\left(f\left(x^{2^{n}}\right)-f(0)\right)
$$

for all $x \in \tilde{G}$.

Proof: Assume that $f \in \mathscr{A}_{(\tilde{G}, E)}^{(\lambda)}$. By Lemma 3, we obtain $\mathscr{F}_{y}^{(1)}(x) \leqslant \mathscr{M}_{\delta}^{\lambda}$ for all $x, y \in \tilde{G}$, i.e.,

$$
\left\|f\left(x y^{-1}\right)-2 f(x)+f(x y)\right\| \leqslant \mathscr{M}_{\delta}^{\lambda} .
$$


We define a function $\tilde{f}: \tilde{G} \rightarrow E$ by $\tilde{f}(x)=f(x)-$ $f(0)$. It can be observed that $\tilde{f}(0)=0$. Then for each $x, y \in \tilde{G}$, we have

$$
\left\|\frac{1}{2}\left(\tilde{f}(x y)+\tilde{f}\left(x y^{-1}\right)\right)-\tilde{f}(x)\right\| \leqslant \frac{1}{2} \mathscr{M}_{\delta}^{\lambda} .
$$

Putting $y=x$, we obtain

$$
\left\|\frac{1}{2} \tilde{f}\left(x^{2}\right)-\tilde{f}(x)\right\| \leqslant \frac{1}{2} \mathscr{M}_{\delta}^{\lambda} .
$$

For each positive integer $n$ and each $x \in \tilde{G}$, we apply (28) to obtain

$$
\begin{aligned}
\left\|\frac{1}{2^{n}} \tilde{f}\left(x^{2^{n}}\right)-\tilde{f}(x)\right\| & =\left\|\sum_{i=1}^{n}\left(\frac{\tilde{f}\left(x^{2^{i}}\right)}{2^{i}}-\frac{\tilde{f}\left(x^{2^{i-1}}\right)}{2^{i-1}}\right)\right\| \\
& \leqslant\left(1-\frac{1}{2^{n}}\right) \mathscr{M}_{\delta}^{\lambda} .
\end{aligned}
$$

Consider the sequence $\left\{2^{-n} f\left(x^{2^{n}}\right)\right\}$. For all positive integers $m, n$ and every $x \in \tilde{G}$, we use (29) to obtain

$$
\begin{aligned}
\left\|\frac{\tilde{f}\left(x^{2^{n+m}}\right)}{2^{n+m}}-\frac{\tilde{f}\left(x^{2^{n}}\right)}{2^{n}}\right\| & =\frac{1}{2^{n}}\left\|\frac{\tilde{f}\left(x^{2^{n} \cdot 2^{m}}\right)}{2^{m}}-\tilde{f}\left(x^{2^{n}}\right)\right\| \\
& \leqslant \frac{1}{2^{n}}\left(1-\frac{1}{2^{m}}\right) \mathscr{M}_{\delta}^{\lambda} .
\end{aligned}
$$

Hence $\left\{2^{-n} f\left(x^{2^{n}}\right)\right\}$ is a Cauchy sequence. We can define a function $\tilde{J}: \tilde{G} \rightarrow E$ by

$$
\tilde{J}(x)=\lim _{n \rightarrow \infty} \frac{\tilde{f}\left(x^{2^{n}}\right)}{2^{n}} .
$$

Replacing $x$ by $x^{2^{n}}$ and $y$ by $y^{2^{n}}$ in (27), we obtain

$$
\left\|\frac{1}{2}\left(\tilde{f}\left(x^{2^{n}} y^{2^{n}}\right)+\tilde{f}\left(x^{2^{n}} y^{-2^{n}}\right)\right)-\tilde{f}\left(x^{2^{n}}\right)\right\| \leqslant \frac{1}{2} \mathscr{M}_{\delta}^{\lambda} .
$$

Next, multiplying (30) by $2^{-n}$ and taking $n \rightarrow \infty$, we obtain

$$
\tilde{J}(x y)+\tilde{J}\left(x y^{-1}\right)-2 \tilde{J}(x)=0 .
$$

From (29), as $n \rightarrow \infty$, we have

$$
\|\tilde{f}(x)-\tilde{J}(x)\| \leqslant \mathscr{M}_{\delta}^{\lambda}
$$

for all $x \in G$. To show the uniqueness of $\tilde{J}$, let $\mathscr{J}: \tilde{G} \rightarrow E$ satisfy $\mathscr{J}(0)=0$ and $\|\tilde{f}(x)-\mathscr{J}(x)\| \leqslant$
$\mathscr{M}_{\delta}^{\lambda}$ for all $x \in \tilde{G}$. For every positive integer $n$, we obtain

$$
\tilde{J}\left(x^{2^{n}}\right)=2^{n} \tilde{J}(x), \quad \mathscr{J}\left(x^{2^{n}}\right)=2^{n} \mathscr{J}(x) .
$$

Hence

$$
\begin{aligned}
& \|\mathscr{J}(x)-\tilde{J}(x)\| \\
& \quad=\left\|\frac{1}{2^{n}}\left(\tilde{J}\left(x^{2^{n}}\right)-\tilde{f}\left(x^{2^{n}}\right)\right)-\frac{1}{2^{n}}\left(\tilde{f}\left(x^{2^{n}}\right)-\mathscr{J}\left(x^{2^{n}}\right)\right)\right\| \\
& \quad \leqslant \frac{1}{2^{n}}\left\|\tilde{f}\left(x^{2^{n}}\right)-\tilde{J}\left(x^{2^{n}}\right)\right\|+\frac{1}{2^{n}}\left\|\tilde{f}\left(x^{2^{n}}\right)-\mathscr{J}\left(x^{2^{n}}\right)\right\| \\
& \quad \leqslant \frac{2}{2^{n}} \mathscr{M}_{\delta}^{\lambda} .
\end{aligned}
$$

As $n \rightarrow \infty$ in (31), we have $\mathscr{J}(x)=\tilde{J}(x)$ for all $x \in \tilde{G}$. By defining a function $J: \tilde{G} \rightarrow E$ by $J(x)=$ $\tilde{J}(x)+f(0)$ for all $x \in \tilde{G}$, the proof is complete.

\section{REFERENCES}

1. Kannappan PL, Kuczma M (1974) On a functional equation related to the Cauchy equation. Ann Polon Math 30, 49-55.

2. Ger R (1977) On an alternative functional equation. Aequationes Mathematicae 15, 145-162.

3. Forti GL (1979) La soluzione generale dell'equazione funzionale $\{c f(x+y)-a f(x)-b f(y)-d\}\{f(x+$ $y)-f(x)-f(y)\}=0$. Matematiche (Catania) 34, 219-242.

4. Nakmahachalasint P (2012) An alternative Jensen's functional equation on semigroups. ScienceAsia 38, 408-413.

5. Ng CT (1990) Jensen's functional equation on groups. Aequationes Mathematicae 39, 85-99.

6. Parnami JC, Vasudeva HL (1992) On Jensen's functional equation. Aequationes Mathematicae 43, 211-218.

7. Nakmahachalasint P (2013) Stability of an alternative Jensen's functional equation. ScienceAsia 39, 643-648.

8. Srisawat C, Kitisin N, Nakmahachalasint P (2015) On an alternative functional equation of Jensen type on groups. ScienceAsia 41, 280-288.

9. Kominek Z (1989) On a local stability of the Jensen functional equation. Demonstratio Math 22, 499-507.

10. Jung SM (1998) Hyers-Ulam-Rassias stability of Jensen's equation and its application. Proc Am Math Soc 126, 3137-3143. 